\title{
Direct interaction studies between Aspergillus fumigatus and human immune cells; what have we learned about pathogenicity and host immunity?
}

\author{
Charles O. Morton ${ }^{1}$, Maria Bouzani ${ }^{2}$, Juergen Loeffler ${ }^{2}$ and Thomas R. Rogers ${ }^{3}$ \\ 1 School of Science and Health, University of Western Sydney, Campbelltown, NSW, Australia \\ ${ }_{2}^{2}$ Medizinische Klinik und Poliklinik II, Universitätsklinikum Würzburg, Würzburg, Germany \\ ${ }^{3}$ Department of Clinical Microbiology, Trinity College Dublin, Sir Patrick Dun Research Laboratory, St James's Hospital, Dublin, Ireland
}

\section{Edited by:}

Elaine M. Bignell, Imperial College

London, UK

\section{Reviewed by:}

Dominique Sanglard, University of Lausanne and University Hospital

Center, Switzerland

Sven Krappmann, University Hospital

Erlangen,

Friedrich-Alexander-Universität

Erlangen-Nürnberg, Germany

\section{${ }^{*}$ Correspondence:}

Charles O. Morton, School of Science and Health, University of Western

Sydney, Campbelltown, NSW

2560, Australia.

e-mail:o.morton@uws.edu.au
Invasive aspergillosis is a significant threat to health and is a major cause of mortality in immunocompromised individuals. Understanding the interaction between the fungus and the immune system is important in determining how the immunocompetent host remains disease free. Several studies examining the direct interaction between Aspergillus fumigatus and purified innate immune cells have been conducted to measure the responses of both the host cells and the pathogen. It has been revealed that innate immune cells have different modes of action ranging from effective fungal killing by neutrophils to the less aggressive response of dendritic cells. Natural killer cells do not phagocytose the fungus unlike the other innate immune cells mentioned but appear to mediate their antifungal effect through the release of gamma interferon. Transcriptional analysis of $A$. fumigatus interacting with these cells has indicated that it can adapt to the harsh microenvironment of the phagosome and produces toxins, ribotoxin and gliotoxin, that can induce cell death in the majority of innate immune cells. These data point toward potential novel antifungal treatments including the use of innate immune cells as antifungal vaccines.

Keywords: Aspergillus, innate immunity and responses, host-pathogen interactions, aspergillosis, cellular immunity

\section{INTRODUCTION}

The fungus Aspergillus fumigatus (teleomorph Neosartorya fumigata; O'Gorman etal., 2009) normally plays a role in the decay of plant organic matter in the environment (Gugnani, 2003). It is also an opportunistic pathogen that causes a spectrum of diseases in humans ranging from allergic reactions to life-threatening invasive disease in immunocompromised individuals (Neofytos et al., 2009). Invasive aspergillosis (IA) is the most severe disease caused by the fungus and is the leading cause of mycosis-related mortality in the immunocompromised (McCormick et al., 2010b).

Invasive aspergillosis is usually caused by the inhalation of asexual spores (conidia) into the alveoli; it is estimated that 200-300 conidia are inhaled daily (Latge, 1999). Conidia are covered in a layer of hydrophobin which is inert to the immune system (Aimanianda etal., 2009) and the presence of dihydroxynaphthalene (DHN)-melanin in the cell wall interferes with host endocytosis (Thywissen et al., 2011) leading to potential long-term survival of inhaled resting conidia. Proteomic analysis of resting conidia indicated the presence of enzymes that allow rapid adaptation to the environment in which the fungus germinates and the establishment of infection in the absence of competent antifungal defenses (Teutschbein et al., 2010). The cellular innate immune system usually provides protection against IA by neutralizing germinating conidia (Hasenberg et al., 2011).

Immunity to fungal infection requires the concerted activity of the cells of the immune system (Figure 1). The importance of alveolar macrophages and neutrophils has been the subject of relatively extensive research and will not be the major focus of this review. We will primarily focus on dendritic cells (DC), monocytes, and the emerging role of natural killer (NK) cells in immunity to IA; examining how these analyses have added to the understanding of fungal virulence and immunity to IA.

\section{INTERACTIONS WITH INNATE IMMUNE CELLS}

The first immune cells to interact with germinating fungal spores are the alveolar macrophages and neutrophils that are recruited to the site of infection. These cells recognize pathogen-associated molecular patterns (PAMPs), e.g., $\beta$-1,3-glucan, galactomannan, and chitin, that are exposed on the surface of germinating conidia or hyphae through pathogen-recognition receptors (PRR) such as Toll-like receptors (TLR; Netea et al., 2003) and Dectin-1 (Brown, 2006). It has been observed in murine models that neutrophils play an essential role in defense against germinating conidia (Mircescu et al., 2009; Ibrahim-Granet et al., 2010). Interference with the innate immune response is a primary factor in the initiation of IA; neutropenia is a primary risk factor for IA (De Pauw et al., 2008). However, increasing evidence suggests that neutrophils and macrophages act cooperatively to defend against infection (Silva, 2011).

\section{MACROPHAGES}

The population of resident alveolar macrophages (AM) are thought to be the first immune cells to encounter A. fumigatus in the lung (Figure 1). The interaction between A. fumigatus and 


\section{(1) Macrophages}

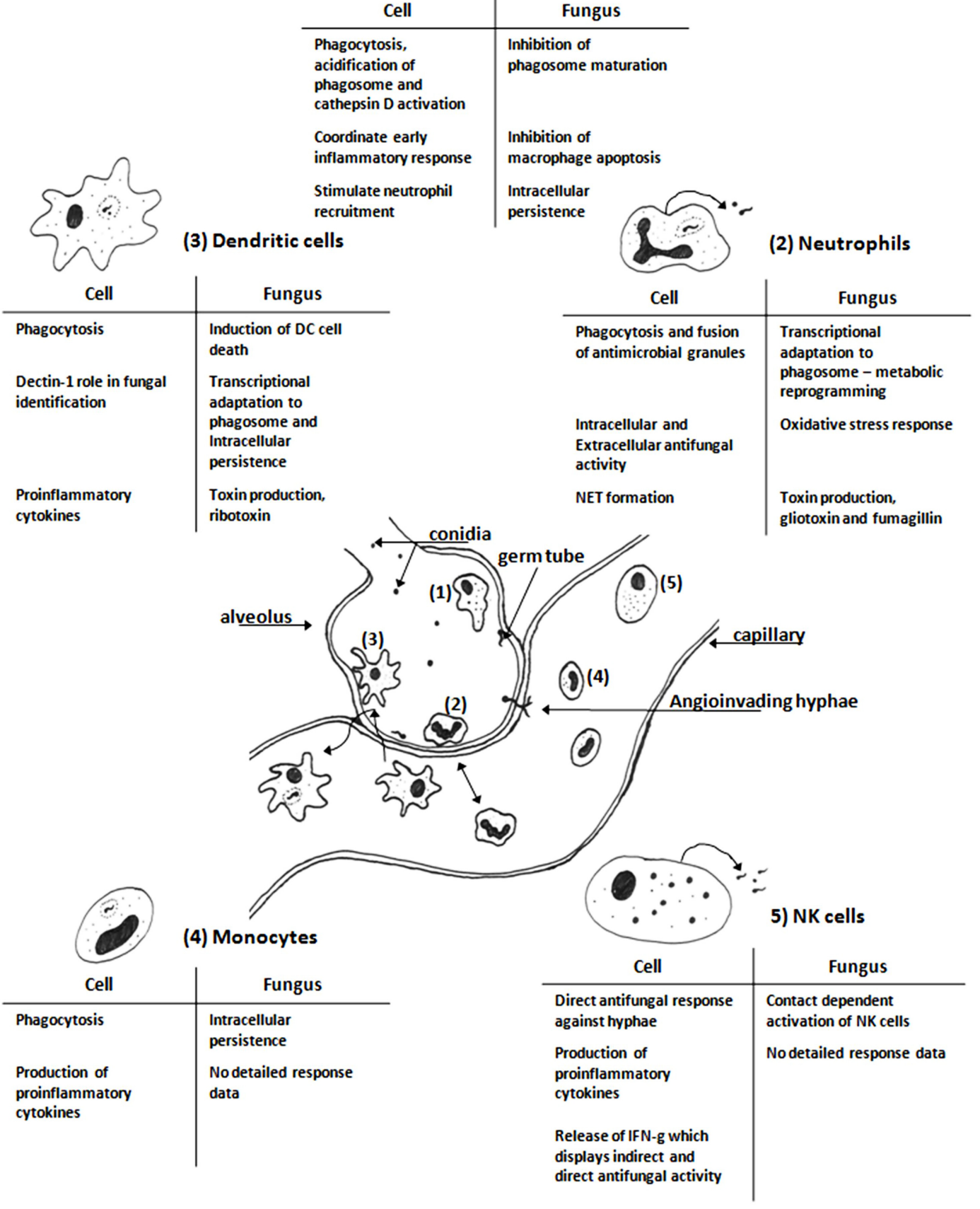

FIGURE 1 | Schematic diagram of cells of the innate immune system interacting with Aspergillus fumigatus in the vicinity of an alveolus. The resident alveolar macrophages (1) are first to encounter the fungus and after phagocytosis they kill the ingested fungus and initiate the inflammatory response (Segal, 2007; Volling et al., 2007; Slesiona et al., 2012). Neutrophils (2) are recruited to the site of infection by chemoattractant cytokines, they are necessary for clearance of the fungal infection (Sugui et al., 2008; Brakhage et al., 2010; Fallon et al., 2010). Dendritic cells (3) actively scan the body for potential infectious agents; they phagocytose pathogens and drain to the lymph nodes where they can present antigens from the degraded pathogen to induce an adaptive immune response (Bozza et al., 2002; Mezger et al., 2008; Morton et al., 2011). Monocytes, progenitors for macrophages and some dendritic cell, (4) are present in blood; they can phagocytose fungal elements and release proinflammatory cytokines (Rodland et al., 2008; Loeffler et al., 2009). NK cells (5) are present in blood and can be attracted to sites of infection; they release antimicrobial molecules that can directly kill invading fungi (Park et al., 2009; Bouzani et al., 2011). The tables are summaries of the key features of the interactions between $A$. fumigatus with each immune cell based on the studies cited in each section. The figure is not to scale. 
macrophages has provided an important model for the studying phagocytosis of the fungus. Conidia are phagocytosed by AM and killed following acidification of the phagolysosome and activation of antimicrobial enzymes (e.g., cathepsin D and chitinase; Ibrahim-Granet et al., 2003). Reactive oxygen species (ROS) are required to kill conidia but are not directly responsible for killing the fungus (Philippe et al., 2003; Cornish et al., 2008). AM along with direct antifungal activity also release cytokines to initiate an inflammatory response to fungal infection (Agarwal et al., 2007).

Fungal survival during interactions with AM is associated with fungal DHN-melanin inhibiting acidification of the phagolysosome (Thywissen et al., 2011). This promotes germination of the conidia and escape from the AM through hyphal growth (Slesiona et al., 2012). Another factor that is important for fungal survival during encounters with AM is the production of siderophores, deletion of genes encoding these iron scavenging molecules decreased fungal survival within the AM phagolysosome (Schrettl et al., 2010).

\section{NEUTROPHILS}

Neutrophils are recruited to the site of infection by cytokines, primarily IL-8 in the lung, and are important players in the inflammatory response associated with the clearance of fungal infection (Bellocchio et al., 2004; Bonnett et al., 2006; Park and Mehrad, 2009). These cells recognize fungi through PRR-PAMP interactions and can phagocytose pathogenic microbes. Neutrophils are also capable of attacking hyphae through the production of neutrophil extracellular traps (NET) which are formed when neutrophils undergo autolysis releasing their DNA into the surrounding environment to impede the progress of infection (Bruns et al., 2010). NET formation is important in inhibition of hyphal growth and their formation can be induced by both conidia and hyphae (McCormick et al., 2010a). Interestingly, restoration of NET formation in neutrophils from a patient with chronic granulomatous disease (CGD) restored resistance to IA (Bianchi et al., 2011).

Transcriptional analysis of $A$. fumigatus that is directly interacting with neutrophils has been conducted and has revealed that there is a complex response by genes involved in oxidative stress and fatty acid catabolism (Sugui et al., 2008). Up-regulation of catalases (cat1 and cat2), superoxide dismutase (Mn-SOD), and thioredoxin reductase are consistent with a reaction to oxidative stress caused by the generation of ROS within the phagolysosome in neutrophils.

There was a shift in the metabolism of the fungus to a state similar to that observed under glucose limitation indicating that phagocytosis by neutrophils creates nutrient limiting conditions (Sugui et al., 2008). Transcriptome analysis of A. fumigatus initiating infection in the murine lung revealed that nutrient limitation could be a cue for a trophic switch requiring the induction of putative virulence genes (McDonagh et al., 2008). This behavior has been seen in fungi developed as biological control agents where the absence of nutrients induced the expression of serine proteases that acted as virulence factors against invertebrates (St Leger et al., 1987; Segers et al., 1994). Up-regulation of secondary metabolite gene clusters including the gliotoxin gene cluster during infection of the murine lung may be important for the interaction with neutrophils since gliotoxin has been shown to inhibit phagocytosis and induce apoptosis of neutrophils (Comera et al., 2007).

\section{INTERACTIONS WITH ANTIGEN-PRESENTING CELLS}

The inflammatory response initiated by AM and neutrophils also attracts antigen-presenting cells (APC) from the blood and surrounding tissues through the activity of cytokines (Burns and Thrasher, 2004) and alarmins, such as defensins (Oppenheim et al., 2007). The primary APC are DCs and monocytes. The APCs exist as a number of distinct subpopulations; monocyte subpopulations are $\mathrm{CD} 14^{+} \mathrm{CD} 16^{-}$and $\mathrm{CD} 14^{+} \mathrm{CD}^{+} 6^{+}$(Serbina et al., 2009). In humans $90 \%$ of circulating monocytes have $\mathrm{CD} 14^{+} \mathrm{CD} 16^{-}$ markers, these can phagocytose conidia and inhibit germination but secrete small amounts of TNF-alpha. However, CD $14^{+}$CD $16^{+}$monocytes, the remaining $10 \%$, do not inhibit germination but secrete large amounts of TNF-alpha (Serbina et al., 2009). Monocytes are precursor cells for specific populations of DC and macrophages (Osugi et al., 2002).

The major subgroups of DC include myeloid DC (mDC), plasmacytoid DC (pDC), and monocyte-derived DC (moDC). Both $\mathrm{mDC}$ and $\mathrm{pDC}$ occur in low numbers within the bloodstream which is why moDC are frequently used in experiments since they can be generated in large numbers. It has been postulated that moDC in the body may represent an auxillary inflammatory pathway whereas $\mathrm{mDC}$ and $\mathrm{pDC}$ are the specialized surveillance subsets of DC (Osugi et al., 2002). APCs link the innate and adaptive arms of the immune response since the antigens induce a pathogen-specific T helper cell response (Bozza et al., 2002).

\section{DENDRITIC CELLS}

Dendritic cells act as a surveillance system for the body; they can be found in the majority of tissues and the circulatory system in an immature state (Wuthrich et al., 2012). They act by sampling their microenvironment for potential microbial pathogens, uptake of a microbe leads to DC maturation and the presentation of microbial antigens in the lymph nodes activates specific T cells. These cells drive the adaptive immune system to produce a Th-1 or Th- 2 type response; the inflammatory Th-1 response is associated with the clearance of fungal infection.

Whole genome analyses of the interaction between immature moDC, from healthy donors, and A. fumigatus have been conducted for both organisms. Analysis of the moDC transcriptome revealed that exposure to A. fumigatus induced a proinflammatory response and indicated the importance of Dectin-1 in fungal recognition. Among the genes that were up-regulated were CCL20, IL1B,IL8, and PTX-3. The expression of SYK and IL2RA were considered indicative of a role for DC in the activation of NK cells (Mezger et al., 2008). This expression pattern was confirmed by transcriptional analysis using a microarray of 120 immune-related genes which also showed the induction of a pro-inflammatory response of moDC to A. fumigatus (Morton et al., 2011).

Direct interaction between moDC and A. fumigatus leads to rapid phagocytosis of the fungal cells with phagocytosis of $48 \%$ of conidia after $2 \mathrm{~h}$ (Bozza et al., 2002) and 68\% after $3 \mathrm{~h}$ (Morton et al., 2011). As mentioned with fungal survival in macrophages, 
germ tubes of A. fumigatus emerged from moDC after $6 \mathrm{~h}$, which coincided with an increase in moDC cell death. Whole genome transcriptome analysis of $A$. fumigatus interacting with immature DC (iDC) at four time points over $12 \mathrm{~h}$ identified 210 differentially regulated genes which showed significant up-regulation of genes involved in fermentation, drug transport, pathogenesis, and response to oxidative stress (Morton et al., 2011). It was interesting that catalases were not up-regulated by the A. fumigatus during interaction with moDC, this contrasts with the data from interactions with neutrophils. This occurs because DCs do not acidify the phagolysosome; this is achieved by tightly controlling ROS generation and contributes to antigen preservation (Watts, 2006).

In contrast to the transcription response in murine lungs there was no differential regulation of the gliotoxin gene cluster. However, Aspf1 was up-regulated as germ tubes emerged after $9 \mathrm{~h}$ co-incubation, which corresponded with an increase in CCL20 expression in the iDC. The allergen Aspf1 is a fungal ribotoxin; a class of RNases that cleave RNA in ribosomes leading to disrupted protein synthesis and apoptosis in target cells (Lacadena et al., 2007). CCL20 expression had previously been linked to Aspf1 in an experiment where moDC were co-incubated with purified Aspf1 (Ok et al., 2009). CCL20 is associated with allergic, Th2, responses in conditions such as asthma (Reibman et al., 2003). As an allergen Aspf1 interacts with moDC to induce an allergic response, characterized by CCL20 expression, which is seen in allergic bronchopulmonary aspergillosis (ABPA; Lacadena et al., 2007).

It has been reported that $\mathrm{pDC}$ were able to inhibit the growth of A. fumigatus hyphae through contact-independent cytotoxicity (Ramirez-Ortiz et al., 2011). This study indicated a close resemblance between the antifungal activities of pDC and NK cells (Bouzani et al., 2011).

\section{MONOCYTES}

Monocytes originate from the bone marrow and, in response to certain immunological cues, such as IA, they migrate via the circulation to the lungs; where they differentiate into DC (Cramer et al., 2011). In mice, Ly6 $\mathrm{C}^{\text {hi }}$ monocytes express the chemokine receptor CCR2 which appears to influence their migration from bone marrow into the circulation (Serbina and Pamer, 2006). In the steady state, circulating CCR2 ${ }^{+}$Ly6C $^{\text {hi }}$ cells differentiate into CD $103^{+}$DCs while CCR2 Ly6C ${ }^{\text {lo }}$ cells differentiate into CD11b ${ }^{+}$DCs (Jakubzick et al., 2008). However, in experimental pulmonary Aspergillus infection CCR2 ${ }^{+} \mathrm{Ly}_{6 \mathrm{C}}{ }^{\mathrm{HI}}$ cells differentiate into $\mathrm{CD} 11 \mathrm{~b}^{+}$DCs. This results in phagocytosis of fungal conidia through recognition of $\beta$ - D-glucan by Dectin- 1 . Although monocytes can inhibit fungal growth it is controversial as to whether they can actually kill Aspergillus. Monocytes are also APCs, and through the PRR's TLR, pentraxin 3, and C type lectins, they can trigger adaptive $\mathrm{T}$ cell responses.

The transcriptional responses of healthy human donor monocytes to A. fumigatus have been the subject of comparable studies. The response of donor monocytes to A. fumigatus conidia measured over 1800 genes differentially expressed when compared to unstimulated control monocytes (Cortez et al., 2006), while over 400 genes were expressed solely in response to A. fumigatus conidia compared to monocytes stimulated with lipopolysaccharide (LPS; Rodland et al., 2008). In the study of Cortez et al. (2006), over $80 \%$ of monocytes had phagocytosed conidia which was comparable to the results for moDC (Morton et al., 2011). The expression of cytokine receptor encoding genes IL1 and IL10 progressively increased over the $6 \mathrm{~h}$ time course as did the expression of chemokine receptor genes CXCL2, CCL3, CCL4, and CCL20. Of genes that encode PRRs PTX-3 (long pentraxin 3) expression also increased over this time course. Interestingly, there was downregulation of TLR1 and no change in expression of either TLR2 or TLR4 (Cortez et al., 2006). The study from Rodland et al. (2008) differed in that a significant increase in expression of TLR5 was observed. This had not been identified previously as having a role in host defense against moulds; it was hypothesized that this may suggest a role for regulatory $T$ cells in immunity to IA. By contrast, there was no up-regulation of other TLR gene expression; however they did observe up-regulation of IRF8 and IRAK1 expression. Interferon regulating factors are transcription factors involved in pro-inflammatory cytokine responses. Another potentially important finding was production of anti-apoptotic gene responses in A. fumigatus infected monocytes. Further in vitro experiments by this group support a role for TLR5 in immune response to A. fumigatus (Rodland et al., 2011).

Aspergillus fumigatus exists as different morphotypes (conidia and hyphae) during infection, the transcriptional response of donor monocytes to A. fumigatus hyphae has also been measured (Loeffler et al., 2009). After 3 h co-incubation 602 monocyte genes were differentially regulated in response to A. fumigatus hyphae compared to 206 in response to resting conidia. A range of cytokines and chemokines had increased expression in response to A. fumigatus conidia and hyphae however there was no differential regulation of either TLR2 or TLR4. In parallel ELISA assays the authors noted increased production of IL-8, CCL2, and CCL20 which together with the finding of increased expression of plasminogen activating genes and PTX-3 might suggest a mechanism for pulmonary thrombosis and local tissue injury at the site of Aspergillus infection.

From the findings of these three transcriptome studies, innate immune molecules, pro-inflammatory cytokines, and immunomodulatory responses appear to be central to the host monocyte response to Aspergillus infection. It would be important to translate these data to the clinical setting by experimentally studying the in vitro capacity of immunosuppressed patients' monocytes to respond to Aspergillus challenge

\section{INTERACTIONS WITH NK CELLS}

Natural killer cells are innate immune lymphocytes that have been extensively studied due to their ability to kill virus infected and transformed cells without any prior immunization (Caligiuri, 2008). They have an immunoregulatory role, which is fulfilled via the release of several cytokines, predominantly interferon- $\gamma$ (IFN$\gamma)$ as well as chemokines and growth factors (Smyth et al., 2005). Upon infection or inflammation, NK cells migrate from the blood to the lungs, where they become activated (Culley, 2009). During infection by non-viral pathogens regulation of NK cell function is relatively indirect, resulting from signals delivered by accessory cells (Newman and Riley, 2007), but there is growing evidence 
of a direct interplay between NK cells and pathogenic microbes, including bacteria (Sporri etal., 2006), parasites (Hansen et al., 2007), and yeast (Ma et al., 2004). The interaction between NK cells and A. fumigatus is a particularly interesting and relatively unexplored field.

The first demonstration of a role for NK cells in the host defense against IA was achieved in a neutropenic mouse model of IA. The early influx of NK cells into the lungs of mice with IA was linked to an increase in pulmonary CCL2; neutralization of CCL2 impaired the recruitment of NK cells and led to increased mortality (Morrison et al., 2003). A similar experimental model indicated that IFN- $\gamma$, produced in the lungs by NK cells, triggers antifungal mechanisms and mediates the protective impact of NK cells (Park etal., 2009). This indicated that NK cell-derived IFN- $\gamma$ was the mediator of the NK cell protective effect against IA, and excluded the involvement of NK cell cytotoxic proteins (e.g., perforin, granzymes, granulysin). NK cell-derived IFN- $\gamma$ also increased the capacity of macrophages to inhibit the germination of conidia. These findings suggested that NK cells are a critical component of the innate immune response against IA.

More recent studies on the interaction between purified human NK cells and A. fumigatus (Bouzani et al., 2011; Schmidt et al., 2011) have both confirmed the ability of NK cells to mount an effective defense against IA but through contrasting mechanisms of antifungal activity. The debate raised by these contrasting studies has been discussed elsewhere (Bouzani et al., 2012). Schmidt et al. (2011) showed that NK cells with or without interleukin-2 (IL-2) stimulation could kill hyphae but not conidia with perforin acting as the mediator of the cytotoxic mechanism. In contrast, Bouzani et al. (2011) demonstrated a two-step antifungal mechanism, where contact-dependent activation of NK cells by hyphae provoked the release of IFN- $\gamma$ able to damage the fungus. Second, upon its secretion, the NK cell-derived IFN- $\gamma$ was capable of acting against hyphae that were not in physical contact with the NK cells. This mechanism was found to be independent of the degranulation NK cells (release of perforin). IFN- $\gamma$-mediated antifungal activity of NK cells was consistent with the results of the neutropenic mouse model (Park et al., 2009). The pathway through which IFN- $\gamma$ attacks $A$. fumigatus remains to be elucidated.

The anti-Aspergillus activity of invariant natural killer T (iNKT) has also been studied (Cohen et al., 2011). In an immunocompetent mouse model, it was shown that $A$. fumigatus activated iNKT cells in the presence of CD1d ${ }^{+}$APC. Instead of lipids it was $\beta-1,3$ glucan that induced the release of IL-12 by APCs and thereafter the activation of iNKT cells to secrete IFN- $\gamma$.

\section{REFERENCES}

Agarwal, R., Whang, D. H., Alvero, A. B., Visintin, I., Lai, Y., Segal, E. A., et al. (2007). Macrophage migration inhibitory factor expression in ovarian cancer. Am. J. Obstet. Gynecol. 196, 348.e341-e345.

Aimanianda, V., Bayry, J., Bozza, S., Kniemeyer, O., Perruccio, K., Elluru, S. R., etal. (2009). Surface hydrophobin prevents immune

\section{VACCINE AND THERAPEUTIC DEVELOPMENT}

The strong evidence, provided by the studies mentioned in this review, for the ability of immune cells to control the growth of A. fumigatus in vitro has suggested the possibility of using innate immune cells as vaccines against IA. The ability of purified A. fumigatus antigens to modulate the immunity of iDC (Ok et al., 2009) and the capacity of Aspergillus-pulsed DC to drive a Th1 host immune response in mice (Bozza et al., 2003) have indicated the vaccination potential of DC (Roy and Klein, 2012). The transfer of NK cells to immunosuppressed patients to generate a protective response against IA is also possible. To date, existing reports show a protective effect of NK cell transfer to animal hosts with IA (Park et al., 2009). However, the promising evidence is counter-balanced by the limited number of studies, the potential side effects and the unknown characteristics of the candidate treatment group.

The in vitro interactions of monocytes and Aspergillus with the antifungal drugs voriconazole or lipid formulations of amphotericin B have been investigated. The results suggest that under the experimental conditions used these drugs can promote proinflammatory immune responses of monocytes to A. fumigatus hyphae (Simitsopoulou et al., 2008, 2011), suggesting a possible role in clearing the fungus in vivo. The use of exogenous IFN $-\gamma$ as an immunological adjunct to antifungal therapy has been found to be effective in individual renal transplant cases with A. fumigatus pulmonary infection (Armstrong-James et al., 2010). The likely mechanism is by IFN $-\gamma$ immunotherapy enhancing the ability of pulmonary phagocytic cells to promote pro-inflammatory responses in Aspergillus infection which facilitates clearance of the fungus.

\section{CONCLUSION}

Studies of the interactions between A. fumigatus and immune cells have contributed greatly to the understanding of the hostpathogen interaction during aspergillosis (Figure 1). These have indicated that under in vitro conditions the fungus can survive interactions with macrophages and DC through its ability to adapt to the harsh environmental conditions within the host rather than a defined pathology reliant on specific virulence factors. In identifying the roles of lesser studied immune cells in defense against IA it has been possible to identify novel therapeutic strategies that may eventually ease the burden of IA.

\section{ACKNOWLEDGMENTS}

The authors would like to thank the European Science Foundation for funding the ESF Fuminomics consortium that enabled the collaboration for this article.

S., Montagnoli, C., etal. (2004). TLRs govern neutrophil activity in aspergillosis. J. Immunol. 173, 74067415.

Bianchi, M., Niemiec, M. J., Siler, U., Urban, C. F., and Reichenbach, J. (2011). Restoration of antiAspergillus defense by neutrophil extracellular traps in human chronic granulomatous disease after gene therapy is calprotectin-dependent. $J$.
Allergy Clin. Immunol. 127, 12431252.e1247.

Bonnett, C. R., Cornish, E. J., Harmsen, A. G., and Burritt, J. B. (2006). Early neutrophil recruitment and aggregation in the murine lung inhibit germination of Aspergillus fumigatus conidia. Infect. Immun. 74, 65286539.

Bouzani, M., Einsele, H., and Loeffler, J. (2012). Functional analysis is 
a paramount prerequisite for understanding the in vitro interaction of human natural killer cells with Aspergillus fumigatus. J. Infect. Dis. 205, 1025-1026; author reply 10261027.

Bouzani, M., Ok, M., Mccormick, A., Ebel, F., Kurzai, O., Morton, C. O., et al. (2011). Human NK cells display important antifungal activity against Aspergillus fumigatus, which is directly mediated by IFN-gamma release. J. Immunol. 187, 13691376.

Bozza, S., Gaziano, R., Spreca, A., Bacci, A., Montagnoli, C., Di Francesco, P. et al. (2002). Dendritic cells transport conidia and hyphae of Aspergillus fumigatus from the airways to the draining lymph nodes and initiate disparate Th responses to the fungus. J. Immunol. 168, 1362-1371.

Bozza, S., Perruccio, K., Montagnoli, C., Gaziano, R., Bellocchio, S., Burchielli, E., etal. (2003). A dendritic cell vaccine against invasive aspergillosis in allogeneic hematopoietic transplantation. Blood 102, 38073814.

Brakhage, A. A., Bruns, S., Thywissen, A., Zipfel, P. F., and Behnsen, J. (2010). Interaction of phagocytes with filamentous fungi. Curr. Opin. Microbiol. 13, 409-415.

Brown, G. D. (2006). Dectin-1: a signalling non-TLR pattern-recognition receptor. Nat. Rev. Immunol. 6, 33-43.

Bruns, S., Kniemeyer, O., Hasenberg, M., Aimanianda, V., Nietzsche, S., Thywissen, A., et al. (2010). Production of extracellular traps against Aspergillus fumigatus in vitro and in infected lung tissue is dependent on invading neutrophils and influenced by hydrophobin RodA. PLoS Pathog. 6, e1000873. doi: 10.1371/journal.ppat. 1000873

Burns, S., and Thrasher, A. J. (2004). Dendritic cells: the bare bones of immunity. Curr. Biol. 14, R965R967.

Caligiuri, M. A. (2008). Human natural killer cells. Blood 112, 461-469.

Cohen, N. R., Tatituri, R. V., Rivera, A., Watts, G. F., Kim, E. Y., Chiba, A., et al. (2011). Innate recognition of cell wall beta-glucans drives invariant natural killer $\mathrm{T}$ cell responses against fungi. Cell Host Microbe 10, 437-450.

Comera, C., Andre, K., Laffitte, J., Collet, X., Galtier, P., and MaridonneauParini, I. (2007). Gliotoxin from Aspergillus fumigatus affects phagocytosis and the organization of the actin cytoskeleton by distinct signalling pathways in human neutrophils. Microbes Infect. 9, 47-54.
Cornish, E. J., Hurtgen, B. J., Mcinnerney, K., Burritt, N. L., Taylor, R. M., Jarvis, J. N., et al. (2008). Reduced nicotinamide adenine dinucleotide phosphate oxidaseindependent resistance to Aspergillus fumigatus in alveolar macrophages. J. Immunol. 180, 6854-6867.

Cortez, K. J., Lyman, C. A., Kottilil, S., Kim, H. S., Roilides, E., Yang, J., et al. (2006). Functional genomics of innate host defense molecules in normal human monocytes in response to Aspergillus fumigatus. Infect. Immun. 74, 2353-2365.

Cramer, R. A., Rivera, A., and Hohl, T. M. (2011). Immune responses against Aspergillus fumigatus: what have we learned? Curr. Opin. Infect. Dis. 24, 315-322.

Culley, F. J. (2009). Natural killer cells in infection and inflammation of the lung. Immunology 128, 151-163.

De Pauw, B., Walsh, T. J., Donnelly, J. P., Stevens, D. A., Edwards, J. E., Calandra, T., et al. (2008). Revised definitions of invasive fungal disease from the European Organization for Research and Treatment of Cancer/Invasive Fungal Infections Cooperative Group and the National Institute of Allergy and Infectious Diseases Mycoses Study Group (EORTC/MSG) Consensus Group. Clin. Infect. Dis. 46, 18131821.

Fallon, J. P., Reeves, E. P., and Kavanagh, K. (2010). Inhibition of neutrophil function following exposure to the Aspergillus fumigatus toxin fumagillin. J. Med. Microbiol. 59, 625-633.

Gugnani, H. C. (2003). Ecology and taxonomy of pathogenic aspergilli. Front. Biosci. 8, s346-s357.

Hansen, D. S., Bernard, N. J., Nie, C. Q., and Schofield, L. (2007). NK cells stimulate recruitment of CXCR3 $+\mathrm{T}$ cells to the brain during Plasmodium berghei-mediated cerebral malaria. J. Immunol. 178, 5779-5788.

Hasenberg, M., Behnsen, J., Krappmann, S., Brakhage, A., and Gunzer, M. (2011). Phagocyte responses towards Aspergillus fumigatus. Int. J. Med. Microbiol. 301, 436-444.

Ibrahim-Granet, O., Jouvion, G., Hohl, T. M., Droin-Bergere, S., Philippart, F., Kim, O. Y., et al. (2010). In vivo bioluminescence imaging and histopathopathologic analysis reveal distinct roles for resident and recruited immune effector cells in defense against invasive aspergillosis. BMC Microbiol. 10, 105. doi: 10.1186/1471-2180-10-105

Ibrahim-Granet, O., Philippe, B., Boleti, H., Boisvieux-Ulrich, E., Grenet, D., Stern, M., et al. (2003).
Phagocytosis and intracellular fate of Aspergillus fumigatus conidia in alveolar macrophages. Infect. Immun. 71 891-903.

Jakubzick, C., Tacke, F., Ginhoux, F., Wagers, A. J., Van Rooijen, N., Mack, M., etal. (2008). Blood monocyte subsets differentially give rise to $\mathrm{CD} 103+$ and $\mathrm{CD} 103$ - pulmonary dendritic cell populations. $J$. Immunol. 180, 3019-3027.

Lacadena, J., Alvarez-Garcia, E., Carreras-Sangra, N., Herrero-Galan, E., Alegre-Cebollada, J., GarciaOrtega, L., etal. (2007). Fungal ribotoxins: molecular dissection of a family of natural killers. FEMS Microbiol. Rev. 31, 212-237.

Latge, J. P. (1999). Aspergillus fumigatus and aspergillosis. Clin. Microbiol. Rev. 12, 310-350.

Loeffler, J., Haddad, Z., Bonin, M., Romeike, N., Mezger, M., Schumacher, U., et al. (2009). Interaction analyses of human monocytes co-cultured with different forms of Aspergillus fumigatus. J. Med. Microbiol. 58, 49-58.

Ma, L. L., Wang, C. L., Neely, G. G., Epelman, S., Krensky, A. M., and Mody, C. H. (2004). NK cells use perforin rather than granulysin for anticryptococcal activity. J. Immunol. 173, 3357-3365.

McCormick, A., Heesemann, L. Wagener, J., Marcos, V., Hartl, D., Loeffler, J., etal. (2010a). NETs formed by human neutrophils inhibit growth of the pathogenic mold Aspergillus fumigatus. Microbes Infect. 12, 928-936.

McCormick, A., Loeffler, J., and Ebel, F. (2010b). Aspergillus fumigatus: contours of an opportunistic human pathogen. Cell. Microbiol. 12, 15351543.

McDonagh, A., Fedorova, N. D., Crabtree, J., Yu, Y., Kim, S., Chen, D., et al. (2008). Sub-telomere directed gene expression during initiation of invasive aspergillosis. PLoS Pathog. 4, e1000154. doi: 10.1371/journal.ppat. 1000154

Mezger, M., Kneitz, S., Wozniok, I., Kurzai, O., Einsele, H., and Loeffler, J. (2008). Proinflammatory response of immature human dendritic cells is mediated by dectin-1 after exposure to Aspergillus fumigatus germ tubes. J. Infect. Dis. 197, 924-931.

Mircescu, M. M., Lipuma, L., Van Rooijen, N., Pamer, E. G., and Hohl, T. M. (2009). Essential role for neutrophils but not alveolar macrophages at early time points following Aspergillus fumigatus infection. J. Infect. Dis. 200, 647-656.
Morrison, B. E., Park, S. J., Mooney, J. M., and Mehrad, B. (2003). Chemokine-mediated recruitment of NK cells is a critical host defense mechanism in invasive aspergillosis. J. Clin. Invest. 112, 1862-1870.

Morton, C. O., Varga, J. J., Hornbach, A., Mezger, M., Sennefelder, H., Kneitz, S., et al. (2011). The temporal dynamics of differential gene expression in Aspergillus fumigatus interacting with human immature dendritic cells in vitro. PLOS ONE 6, e16016. doi: 10.1371/journal.pone.0016016

Neofytos, D., Horn, D., Anaissie, E., Steinbach, W., Olyaei, A., Fishman, J., et al. (2009). Epidemiology and outcome of invasive fungal infection in adult hematopoietic stem cell transplant recipients: analysis of Multicenter Prospective Antifungal Therapy (PATH) Alliance registry. Clin. Infect. Dis. 48, 265-273.

Netea, M. G., Warris, A., Van Der Meer, J. W., Fenton, M. J., VerverJanssen, T. J., Jacobs, L. E., et al. (2003). Aspergillus fumigatus evades immune recognition during germination through loss of toll-like receptor-4-mediated signal transduction. J. Infect. Dis. 188, 320-326.

Newman, K. C., and Riley, E. M. (2007). Whatever turns you on: accessorycell-dependent activation of NK cells by pathogens. Nat. Rev. Immunol. 7, 279-291.

O'Gorman, C. M., Fuller, H. T., and Dyer, P. S. (2009). Discovery of a sexual cycle in the opportunistic fungal pathogen Aspergillus fumigatus. Nature 457, 471-474.

Ok, M., Latge, J. P., Baeuerlein, C., Ebel, F., Mezger, M., Topp, M., et al. (2009). Immune responses of human immature dendritic cells can be modulated by the recombinant Aspergillus fumigatus antigen Aspf1. Clin. Vaccine Immunol. 16, 1485-1492.

Oppenheim, J. J., Tewary, P., De La Rosa, G., and Yang, D. (2007). Alarmins initiate host defense. Adv. Exp. Med. Biol. 601, 185-194.

Osugi, Y., Vuckovic, S., and Hart, D. N. (2002). Myeloid blood CD11c(+) dendritic cells and monocyte-derived dendritic cells differ in their ability to stimulate T lymphocytes. Blood 100, 2858-2866.

Park, S. J., Hughes, M. A., Burdick, M., Strieter, R. M., and Mehrad, B. (2009). Early NK cell-derived IFN\{gamma\} is essential to host defense in neutropenic invasive aspergillosis. J. Immunol. 182, 4306-4312.

Park, S. J., and Mehrad, B. (2009). Innate immunity to Aspergillus species. Clin. Microbiol. Rev. 22, 535-551. 
Philippe, B., Ibrahim-Granet, O., Prevost, M. C., Gougerot-Pocidalo, M. A., Sanchez Perez, M., Van Der Meeren, A., et al. (2003). Killing of Aspergillus fumigatus by alveolar macrophages is mediated by reactive oxidant intermediates. Infect. Immun. 71, 3034-3042.

Ramirez-Ortiz, Z. G., Lee, C. K., Wang, J. P., Boon, L., Specht, C. A., and Levitz, S. M. (2011). A nonredundant role for plasmacytoid dendritic cells in host defense against the human fungal pathogen Aspergillus fumigatus. Cell Host Microbe 9, 415-424.

Reibman, J., Hsu, Y., Chen, L. C., Bleck, B., and Gordon, T. (2003). Airway epithelial cells release MIP-3alpha/CCL20 in response to cytokines and ambient particulate matter. Am. J. Respir. Cell Mol. Biol. $28,648-654$.

Rodland, E. K., Ager-Wick, E., Halvorsen, B., Muller, F., and Froland, S. S. (2011). Toll like receptor 5 (TLR5) may be involved in the immunological response to Aspergillus fumigatus in vitro. Med. Mycol. 49, 375-379.

Rodland, E. K., Mattingsdal, M., Olstad, O. K., Ovstebo, R., Kierulf, P., Muller, F., et al. (2008). Expression of genes in normal human monocytes in response to Aspergillus fumigatus. Med. Mycol. 46, 327-336.

Roy, R. M., and Klein, B. S. (2012). Dendritic cells in antifungal immunity and vaccine design. Cell Host Microbe 11, 436-446.

Schmidt, S., Tramsen, L., Hanisch, M., Latge, J. P., Huenecke, S., Koehl, U., et al. (2011). Human natural killer cells exhibit direct activity against Aspergillus fumigatus hyphae, but not against resting conidia. J. Infect. Dis. 203, 430-435.

Schrettl, M., Ibrahim-Granet, O., Droin, S., Huerre, M., Latge, J. P., and Haas, H. (2010). The crucial role of the Aspergillus fumigatus siderophore system in interaction with alveolar macrophages. Microbes Infect. 12, 1035-1041.

Segal, B. H. (2007). Role of macrophages in host defense against aspergillosis and strategies for immune augmentation. Oncologist 12(Suppl 2), 7-13.

Segers, R., Butt, T. M., Kerry, B. R., and Peberdy, J. F. (1994). The nematophagous fungus Verticillium chlamydosporium produces a chymoelastase-like protease which hydrolyses host nematode proteins in situ. Microbiology 140(Pt 10), 27152723.

Serbina, N. V., Cherny, M., Shi, C., Bleau, S. A., Collins, N. H. Young, J. W., et al. (2009). Distinct responses of human monocyte subsets to Aspergillus fumigatus conidia. J. Immunol. 183, 2678-2687.

Serbina, N. V., and Pamer, E. G. (2006). Monocyte emigration from bone marrow during bacterial infection requires signals mediated by chemokine receptor CCR2. Nat. Immunol. 7, 311-317.

Silva, M. T. (2011). Macrophage phagocytosis of neutrophils at inflammatory/infectious foci: a cooperative mechanism in the control of infection and infectious inflammation. $J$. Leukoc. Biol. 89, 675-683.

Simitsopoulou, M., Roilides, E., Georgiadou, E., Paliogianni, F., and Walsh, T. J. (2011). Differential transcriptional profiles induced by amphotericin B formulations on human monocytes during response to hyphae of Aspergillus fumigatus. Med. Mycol. 49, 176-185.

Simitsopoulou, M., Roilides, E., Paliogianni, F., Likartsis, C., Ioannidis, J., Kanellou, K., et al. (2008). Immunomodulatory effects of voriconazole on monocytes challenged with Aspergillus fumigatus: differential role of Toll-like receptors. Antimicrob. Agents Chemother. 52, 3301-3306.

Slesiona, S., Gressler, M., Mihlan, M., Zaehle, C., Schaller, M., Barz, D., et al. (2012). Persistence versus escape: Aspergillus terreus and Aspergillus fumigatus employ different strategies during interactions with macrophages. PLoS ONE 7, e31223. doi: 10.1371/journal.pone.0031223

Smyth, M. J., Cretney, E., Kelly, J. M., Westwood, J. A., Street, S. E., Yagita, H., et al. (2005). Activation of NK cell cytotoxicity. Mol. Immunol. 42, 501-510.

Sporri, R., Joller, N., Albers, U., Hilbi, H., and Oxenius, A. (2006). MyD88dependent IFN-gamma production by NK cells is key for control of Legionella pneumophila infection. J. Immunol. 176, 6162-6171.

St Leger, R. J., Charnley, A. K., and Cooper, R. M. (1987). Characterization of cuticle-degrading proteases produced by the entomopathogen Metarhizium anisopliae. Arch. Biochem. Biophys. 253, 221-232.

Sugui, J. A., Kim, H. S., Zarember, K. A., Chang, Y. C., Gallin, J. I., Nierman, W. C., et al. (2008). Genes differentially expressed in conidia and hyphae of Aspergillus fumigatus upon exposure to human neutrophils. PLoS ONE 3, e2655. doi: 10.1371/journal.pone. 0002655

Teutschbein, J., Albrecht, D., Potsch, M., Guthke, R., Aimanianda, V. Clavaud, C., et al. (2010). Proteome profiling and functional classification of intracellular proteins from conidia of the human-pathogenic mold Aspergillus fumigatus. J. Proteome Res. 9, 3427-3442.

Thywissen, A., Heinekamp, T., Dahse, H. M., Schmaler-Ripcke, J., Nietzsche, S., Zipfel, P. F., et al..
(2011). Conidial dihydroxynaphthalene melanin of the human pathogenic fungus aspergillus fumigatus interferes with the host endocytosis pathway. Front. Microbiol. 2:96. doi: 10.3389/fmicb.2011.00096

Volling, K., Brakhage, A. A., and Saluz, H. P. (2007). Apoptosis inhibition of alveolar macrophages upon interaction with conidia of Aspergillus fumigatus. FEMS Microbiol. Lett. 275, 250-254.

Watts, C. (2006). Phagosome neutrality in host defense. Cell 126, 17-19.

Wuthrich, M., Deepe, G. S. Jr., and Klein, B. (2012). Adaptive immunity to fungi. Ann. Rev. Immunol. 30, 115-148.

Conflict of Interest Statement: The authors declare that the research was conducted in the absence of any commercial or financial relationships that could be construed as a potential conflict of interest.

Received: 27 July 2012; accepted: 15 November 2012; published online: 03 December 2012.

Citation: Morton CO, Bouzani M, Loeffler J and Rogers TR (2012) Direct interaction studies between Aspergillus fumigatus and human immune cells; what have we learned about pathogenicity and host immunity? Front. Microbio. 3:413. doi: 10.3389/fmicb.2012.00413

This article was submitted to Frontiers in Fungi and Their Interactions, a specialty of Frontiers in Microbiology.

Copyright (C) 2012 Morton, Bouzani, Loeffler and Rogers. This is an openaccess article distributed under the terms of the Creative Commons Attribution License, which permits use, distribution and reproduction in other forums, provided the original authors and source are credited and subject to any copyright notices concerning any third-party graphics etc. 